\title{
Invariant measures for the flow of a first order partial differential equation
}

\author{
R. RUDNICKI \\ Institute of Mathematics, Silesian University 40-007 Katowice, Poland
}

(Received 4 May 1984)

Abstract. We prove that the dynamical systems generated by first order partial differential equations are $K$-flows and chaotic in the sense of Auslander \& Yorke.

\section{0 . Introduction}

The purpose of this paper is to apply a Brownian motion to the problem of the existence of invariant measures for the dynamical systems generated by some first order partial differential equations.

$\S 1$ contains basic notation and definitions. In $\S 2$ we define a flow describing the evolution of solutions of partial differential equations. In the last section we give a construction of an invariant measure for such a flow. This measure is positive on the open sets and non-trivial. The corresponding system is a $K$-flow and the flow is chaotic in the sense of Auslander \& Yorke [1]. These theorems generalize the results of Lasota [5], [6], Brunovský and Komornik [2], [3] and Dawidowicz [4].

\section{Preliminaries}

Let $X$ be a topological Hausdorff space and let $S_{t}: X \rightarrow X, t \in \mathbb{R}$, be a group of transformations. We call the group $\left\{S_{t}\right\}$ a flow if the mapping

$$
\mathbb{R} \times X \ni(t, x) \mapsto S, x \in X
$$

is continuous in $(t, x)$. By a measure on $X$ we mean any probabilistic measure $\mu$ defined on the $\sigma$-algebra $\mathscr{B}(X)$ of Borel subsets of $X$. A measure $\mu$ is called non-trivial with respect to $\left\{S_{t}\right\}$, if $\mu(P)=0$, where $P$ denotes the set of all periodic points of $\left\{S_{t}\right\}$. Let $X$ be a linear topological space, and let $\mu$ be a measure on $X$. We will say that $\mu$ is a Gaussian measure if each continuous linear functional on $X$ has a Gaussian distribution.

Denote by $C^{n}(U, V)$ the space of $n$-times continuously differentiable functions defined on $U$ with values in $V$, where $U$ and $V$ are non-empty intervals. Assume that $C^{n}(U, V)$ is equipped with the topology of uniform convergence (with derivatives of order $\leq n$ ) on compact subsets.

\section{Flow}

Consider the initial value problem

$$
\begin{aligned}
u_{t}+a(x) u_{x} & =b(x, u) & & \text { for }(t, x) \in D, \\
u(0, x) & =v(x) & & \text { for } x \in U_{1} .
\end{aligned}
$$


In this section and throughout the paper we shall assume that $a$ and $b$ are given functions satisfying

(1 $\left.{ }^{\circ}\right) a \in C^{r}\left(\bar{U}_{1}, \mathbb{R}\right)$ for $r \geq 1$;

(2 $2^{\circ} a(x) \neq 0$ for $x \in U_{1}$ and $a(x)=0$ for $x \in \partial U_{1}$;

$\left(3^{\circ}\right)$ there are constants $K$ and $L$ such that

$$
|a(x)| \leq L+K|x| \quad \text { for } x \in U_{1} ;
$$

(4) $b \in C^{r}\left(U_{1} \times \bar{U}_{2}, \mathbb{R}\right)$,

$\left(5^{\circ}\right) b(x, u)=0 \quad$ for $(x, u) \in U_{1} \times \partial U_{2}$,

$\left(6^{\circ}\right)$ there are continuous functions $M(x)$ and $N(x)$ such that

$$
|b(x, u)| \leq M(x)+N(x)|u| \quad \text { for }(x, u) \in U_{1} \times U_{2} \text {. }
$$

Here $U_{1}$ and $U_{2}$ are open intervals (bounded or not) of the real line, $D=\mathbb{R} \times U_{1}$, $\bar{U}_{i}$ denotes the closure of $U_{i}$ in $\mathbb{R}$, and $\partial U_{i}=\vec{U}_{i} \backslash U_{i}$. These conditions will not be repeated in the statements of the theorems.

We denote by $\pi_{t} s$ the unique solution of the equation

$$
x^{\prime}(t)=a(x(t))
$$

with the initial condition $x(0)=s, s \in U_{1}$. By $\psi(t, s, p)$ we denote the solution of the equation

$$
y^{\prime}(t)=b\left(\pi_{t} s, y(t)\right)
$$

with the initial condition $y(0)=p, p \in U_{1}$. From $\left(1^{\circ}\right)-\left(3^{\circ}\right)$ it follows that $\pi$ is defined for all $(t, s) \in \mathbb{R} \times U_{1}$ and possesses $r$ th-order continuous partial derivatives. For given $x_{0} \in U_{1}$ the function $t \mapsto \pi_{t} x_{0}$ is a $C^{r}$-diffeomorphism of $\mathbb{R}$ onto $U_{1}$. From $\left(1^{\circ}\right)-\left(6^{\circ}\right)$ it follows that $\psi$ is a $C^{r}$-mapping of $\mathbb{R} \times U_{1} \times U_{2}$ into $U_{2}$. The functions $\pi$ and $\psi$ satisfy the following equalities

$$
\begin{gathered}
\pi_{s+t} x=\pi_{s}\left(\pi_{t} x\right), \\
\psi(s+t, x, y)=\psi\left(s, \pi_{t} x, \psi(t, x, y)\right)
\end{gathered}
$$

for each $s, t \in \mathbb{R}, x \in U_{1}$ and $y \in U_{2}$. Let $v$ be a continuously differentiable function from $U_{1}$ into $U_{2}$. Then there exists exactly one solution of (E), namely

$$
u(t, x)=\psi\left(t, \pi_{-t} x, v\left(\pi_{-t} x\right)\right)
$$

Let $v$ be a continuous function from $U_{1}$ into $U_{2}$. Then $u(t, x)$ given by the formula (2.3) will be called a generalized solution of (E).

For an integer $n, 0 \leq n \leq r$, we set $X=C^{n}\left(U_{1}, U_{2}\right)$ and $Y=C^{n}(\mathbb{R}, \mathbb{R})$. We shall consider solutions of equation $(E)$ as the trajectories of the flow $\left\{S_{t}\right\}_{t \in R}$ defined on $X$ by the formula

$$
\left(S_{t} v\right)(x)=u(t, x)=\psi\left(t, \pi_{-t} x, v\left(\pi_{-t} x\right)\right) .
$$

We now define a mapping $T: \mathbb{R} \times Y \rightarrow Y$ by

$$
\left(T_{t} v\right)(s)=v(s-t) \text {. }
$$

It is clear that $\left\{T_{t}\right\}_{t \in \mathbb{R}}$ is a flow on $Y$.

THEOREM 1. There exists a homeomorphism $Q$ of $X$ onto $Y$ such that $Q \circ S_{t}=T_{t} \circ Q$ for each $t \in \mathbb{R}$. 
In order to prove this theorem we need the following lemma.

LEMMA 2.1. Let $V_{1}, V_{2}, W_{1}$ and $W_{2}$ be open intervals of R. Assume that $f: W_{1} \times V_{2} \rightarrow$ $W_{2}$ and $g: W_{1} \rightarrow V_{1}$ are $C^{n}$-maps, $n \geq 0$. Then the map $P: C^{n}\left(V_{1}, V_{2}\right) \rightarrow C^{n}\left(W_{1}, W_{2}\right)$ defined by $P(v)(x)=f(x, v(g(x)))$ is continuous.

The proof of this lemma is simple, so it is omitted.

Proof of theorem 1. Given a point $x_{0} \in U_{1}$. The map $t \rightarrow \pi_{t} x_{0}$ is a $C^{n}$-diffeomorphism of $\mathbb{B}$ onto $U_{1}$. Let $h: U_{1} \rightarrow \mathbb{B}$ be the inverse of $\pi x_{0}$. Then $h$ is a $C^{n}$-diffeomorphism of $\mathbb{R}$ onto $U_{1}$. Let $p$ be a $C^{n}$-diffeomorphism of $\mathbb{R}$ onto $U_{2}$. Define the maps $Q: X \rightarrow Y$ and $N: Y \rightarrow X$ by

$$
(Q v)(s)=p^{-1}\left(\psi\left(-s, \pi_{s} x_{0}, v\left(\pi_{s} x_{0}\right)\right)\right)=p^{-1}\left(\left(S_{-s} v\right)\left(x_{0}\right)\right)
$$

and

$$
(N v)(x)=\psi\left(h(x), x_{0}, p(v(h(x)))\right)
$$

From lemma 2.1 the maps $Q$ and $N$ are continuous. Using (2.1) and (2.2) it is easy to verify that $N \circ Q=Q \circ N=I$. Thus $Q$ is a homeomorphism of $X$ onto $Y$. We verify that $Q \circ S_{t}=T_{t} \circ Q$,

$$
\left(Q \circ S_{t}\right)(v)(x)=p^{-1}\left(\left(S_{-x} \circ S_{t} v\right)\left(x_{0}\right)\right)=(Q v)(x-t)=\left(T_{t} \circ Q\right)(v)(x) .
$$

COROllary 1. The set of all periodic points of $\left\{S_{t}\right\}$ is dense in $X$.

Remark 1. From the definition of $Q$ it follows that for every $s \in \mathbb{R}$ we have $(Q v)(s)=(Q w)(s)$ iff $v\left(\pi_{s} x_{0}\right)=w\left(\pi_{s} x_{0}\right)$.

Examples. (1) Let $b(x, u)=f(x)+g(x) u$ and $U_{2}=\mathbb{R}$. Then $Q v=v_{0}+L v$, where $L$ is a linear isomorphism from $C^{n}\left(U_{1}, \mathbb{R}\right)$ onto $C^{n}(\mathbb{R}, \mathbb{R})$ and $v_{0} \in C^{n}(\mathbb{R}, \mathbb{R})$.

(2) Let $a(x)=x, b(x, u)=\lambda u(1-u), U_{1}=\mathbb{R}^{+}$and $U_{2}=(0,1)$. We take $x_{0}=1$ and $p(u)=e^{u} /\left(1+e^{u}\right)$. Then

$$
(Q v)(s)=\ln v\left(e^{s}\right)-\ln \left[e^{\lambda s}-e^{\lambda s} v\left(e^{s}\right)\right] .
$$

3. Measure on the space $C^{n}(\mathbb{R}, \mathbb{R})$

Let $w_{t}, 0 \leq t<\infty$ be a Brownian motion defined on a probability space $(\Omega, \Sigma, P)$. We may assume that the sample functions of $w_{t}$ are continuous. Set $\xi_{x}^{0}=e^{-x} w_{e^{2 x}}$ for $x \in \mathbb{R}$. Then $\xi_{x}^{0}$ is a stationary Gaussian Markov process with mean value $E \xi_{x}^{0}=0$ and correlation function $E \xi_{x}^{0} \xi_{x+h}^{0}=e^{-|h|}$. The sample functions of $\xi_{x}^{0}$ are continuous. The process $\xi_{x}^{0}$ is not differentiable in the mean. From the law of the iterated logarithm it follows that

$$
\lim _{|x| \rightarrow \infty} \frac{\left|\xi_{x}^{0}\right|}{|x|}=0
$$

with probability 1 . We assume that all sample functions of $\xi_{x}^{0}$ satisfy (3.1). Denote by $H$ the closed linear subspace of $L^{2}(\Omega)$ spanned by all variables $\xi_{x}^{0}, x \in \mathbb{R}$. The joint distribution of the random functions $\zeta_{1}, \ldots, \zeta_{n} \in H$ is Gaușsian. Denote by $\mathscr{F}_{T}$ the $\sigma$-algebra of events generated by the process $w_{t}$ on the set $T$. 
Starting from the random process $\xi_{x}^{0}$ we can define, by induction, some new process. Set for $k \geq 0$

$$
\xi_{x}^{k+1}=\int_{-\infty}^{x} e^{s-x} \xi_{s}^{k} d s
$$

By (3.1) the integral (3.2) exists for every $\omega \in \Omega$ and consequently the sample functions of $\xi_{x}^{n}$ possess $n$ th-order continuous derivatives. The integral (3.2) exists also in the mean and consequently $\xi_{x}^{n}$ is a stationary Gaussian process. Write $\eta_{x}=\xi_{x}^{n}$. Then for every $\omega \in \Omega$ we have

$$
\sum_{k=0}^{n}\left(\begin{array}{l}
n \\
k
\end{array}\right) \eta_{x}^{(k)}=\xi_{x}^{0}
$$

The process $\xi_{x}^{n}$ is not $(n+1)$-times differentiable in the mean.

Lemma 3.1. Let $\eta_{x}=\xi_{x}^{n}, n \geq 1$. The joint distribution of $\left(\eta_{x}, \eta_{x}^{\prime}, \ldots, \eta_{x}^{(n-1)}, \xi_{x}^{0}\right)$ is non-degenerate for every $x$.

Proof. The distribution of $\left(\eta_{x}, \eta_{x}^{\prime}, \ldots, \xi_{x}^{0}\right)$ is Gaussian and it is independent of $x$. Assume that it is degenerate. Then for every $x$

$$
a_{0} \eta_{x}+a_{1} \eta_{x}^{\prime}+\cdots+a_{n-1} \eta_{x}^{(n-1)}+a_{n} \xi_{x}^{0}=0 \quad \text { a.e. }
$$

with at least one $a_{k} \neq 0$. Set $p=\max \left\{k: a_{k} \neq 0\right\}$. The process $a_{0} \eta_{x}+a_{1} \eta_{x}^{\prime}+\cdots+a_{n} \xi_{x}^{0}$ is not $(n+1-p)$-times differentiable. This contradicts the equality (3.4).

Set $Y=C^{n}(\mathbb{R}, \mathbb{R})$ and $\eta_{x}=\xi_{x}^{n}, n \geq 0$. Let $\mathscr{B}(Y)$ be the $\sigma$-algebra of Borel subsets of $Y$. The $\sigma$-algebra $\mathscr{B}(Y)$ is generated by the sets of the form $\{\varphi \in Y:(\varphi(x), \ldots$, $\left.\left.\varphi^{(n)}(x)\right) \in B\right\}$, where $x \in \mathbb{R}$ and $B$ is a Borel subset of $\mathbb{R}^{n+1}$. Thus for every $A \in \mathscr{B}(Y)$ we have $\{\eta \in A\} \in \Sigma$. We obtain a probability measure $\mu$ on $\mathscr{B}(Y)$ by setting $\mu(A)=P(\eta \in A)$ for all $A \in \mathscr{B}(Y)$.

Now we shall investigate the properties of $\mu$. The following known property of Wiener measure will be used in the next lemma:

(3.5) If $\varphi:[0, a] \rightarrow \mathbb{R}$ is a continuous function and $\varphi(0)=0$, then for every $\varepsilon>0$ we have

$$
P\left(\left|w_{t}-\varphi(t)\right|<\varepsilon \text { for } t \in[0, a]\right)>0 .
$$

LEMMA 3.2. $\mu(U)>0$ for each non-empty open set $U$.

Proof. If $n=0$, then the proof follows immediately from (3.5). We assume $n \geq 1$. We may assume, without loss of generality, that $U$ is of the form

$$
U=\left\{\varphi \in C^{n}(\mathbb{R}, \mathbb{R}):\left|\varphi^{(k)}(x)-\varphi_{0}^{(k)}(x)\right|<\varepsilon \text { for } x \in[a, b] \text { and } 0 \leq k \leq n\right\},
$$

where $\varphi_{0} \in C^{n}(\mathbb{R}, \mathbb{R}), \varepsilon>0$ and $a, b \in \mathbb{R}$. According to the definition of $\mu$ we have $\mu(U)=P(A)$, where

$$
A=\left\{\omega \in \Omega:\left|\eta_{x}^{(k)}-\varphi_{0}^{(k)}(x)\right|<\varepsilon \text { for } x \in[a, b] \text { and } 0 \leq k \leq n\right\} .
$$

Let $\psi_{0}$ be a continuous function satisfying the following equation

$$
\sum_{k=0}^{n}\left(\begin{array}{l}
n \\
k
\end{array}\right) \varphi_{0}^{(k)}(x)=e^{-x} \psi_{0}\left(e^{2 x}\right)
$$

Set $\alpha=e^{2 a}$ and $\beta=e^{2 b}$. By (3.3) and from the continuous dependence on the initial values and the parameter it follows that there exists $\delta>0$ such that $A_{\delta} \cap B_{\delta} \subset A$, 
where the sets $A_{\delta}$ and $B_{\delta}$ are given by formulae

$$
\begin{array}{ll}
A_{\delta}=\left\{\omega \in \Omega:\left|\eta_{a}^{(k)}-\varphi_{0}^{(k)}(a)\right|<\delta\right. & \text { for } 0 \leq k \leq n-1\}, \\
B_{\delta}=\left\{\omega \in \Omega:\left|w_{t}-\psi_{0}(t)\right|<\delta\right. & \text { for } t \in[\alpha, \beta]\} .
\end{array}
$$

It is easy to verify that the random variables $\eta_{a}^{(k)}$ are $\mathscr{F}_{\leq \alpha}$-measurable and, therefore, $A_{\delta} \in \mathscr{F}_{\leq \alpha}$. We also have $B_{\delta} \in \mathscr{F}_{\geq \alpha}$. By lemma 3.1 the joint distribution of $\left(\eta_{a}, \ldots\right.$, $\left.\eta_{a}^{(n-1)}, \xi_{a}^{0}\right)$ is Gaussian and non-degenerate. Consequently the joint distribution of $\left(\eta_{a}, \ldots, \eta_{a}^{(n-1)}, w_{\alpha}\right)$ is also non-degenerate. Thus we have $P\left(A \mid \mathscr{F}_{=\alpha}\right)>0$ almost everywhere on $B_{\delta}$. According to (3.5) we have $P\left(B_{\delta}\right)>0$. From the Markov property of Brownian motion it follows that

$$
P\left(A_{\delta} \cap B_{\delta}\right)=\int_{B_{\delta}} P\left(A_{\delta} \mid \mathscr{F}_{=\alpha}\right) d P>0,
$$

which completes the proof.

LEMMA 3.3. The measure $\mu$ is invariant under $\left\{T_{t}\right\}$.

Proof. The invariance of $\mu$ follows directly from the stationarity of the process $\left(\eta_{x}\right.$, $\left.\eta_{x}^{\prime}, \ldots, \eta_{x}^{(n)}\right)$.

LEMMA 3.4. The flow $\left\{T_{t}\right\}$ on $(Y, \mathscr{B}(Y), \mu)$ is a $K$-flow.

Proof. Let $\mathscr{B}_{0}$ be the smallest $\sigma$-algebra containing the sets of the form

$$
A=\left\{\varphi \in C^{n}(\mathbb{R}, \mathbb{R}):\left(\varphi(x), \ldots, \varphi^{(n)}(x)\right) \in B\right\},
$$

where $x \leq 0$ and $B$ is a Borel subset of $\mathbb{R}^{n+1}$. The $\sigma$-algebra $T_{t} \mathscr{B}_{0}$ is the smallest $\sigma$-algebra containing the sets of the form (3.6) with $x \leq t$. Thus $\mathscr{B}_{0} \subset T_{t} \mathscr{B}_{0}$ for $t>0$, and $\mathscr{B}(Y)$ is the smallest $\sigma$-algebra containing all the $\sigma$-algebras $T_{t} \mathscr{B}_{0}$ for $t \in \mathbb{R}$. It remains to verify that the $\sigma$-algebra $\bigcap_{t \in \mathbb{R}} T_{t} \mathscr{B}_{0}$ contains only sets of measure zero or one. Let $A \in \bigcap_{t} T_{t} \mathscr{B}_{0}$. Now we define $E$ by $E=\{\omega \in \Omega: \eta .(\omega) \in A\}$. Then $\mu(A)=$ $P(E)$ and $E \in \cap, \mathscr{A}_{\leq t}$, where $\mathscr{A}_{\leq t}$ is the smallest $\sigma$-algebra generated by the random

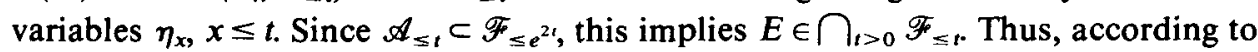
the Blumenthal's zero-or-one law, $P(E)$ is one or zero. This completes the proof.

\section{LEMMA 3.5. $\mu$ is a Gaussian measure.}

Proof. The map $L$ defined by $L(\varphi)=\left(\varphi^{(n)}, \varphi(0), \varphi^{\prime}(0), \ldots, \varphi^{(n-1)}(0)\right)$ is a linear isomorphism between $C^{n}(\mathbb{R}, \mathbb{R})$ and $C^{0}(\mathbb{R}, \mathbb{R}) \times \mathbb{R}^{n}$. From this, and by the Riesz representation theorem every continuous linear functional defined on $C^{n}(\mathbb{R}, \mathbb{R})$ is of the form

$$
f(\varphi)=\int_{a}^{b} \varphi^{(n)}(x) d g(x)+c_{0} \varphi(a)+\cdots+c_{n-1} \varphi^{(n-1)}(a)
$$

where $g$ is a function of bounded variation defined on some interval $[a, b]$, and $\left\{c_{0}, \ldots, c_{n-1}\right\}$ is a sequence of real numbers. From the definition of $\mu$ it follows that $f$ has the same distribution as the random variable $\zeta$ defined by

$$
\zeta=\int_{a}^{b} \eta_{x}^{(n)} d g(x)+c_{0} \eta_{a}+\cdots+c_{n-1} \eta_{a}^{(n-1)}
$$


The random variable $\zeta$ belongs to $H$, thus $\zeta$ has a Gaussian distribution. This completes the proof.

LEMMA 3.6. The measure $\mu$ is non-trivial.

Proof. We denote by $P(t)$ the set of all periodic points of $\left\{T_{t}\right\}$ with period $t$ $\left(P(0)\right.$ denotes the set of all stationary points of $\left.\left\{T_{t}\right\}\right)$. Let $P(\leq t)$ be the set of all periodic points with period $s \in(0, t]$. Then $P(t)$ and $P(\leq t)$ are closed invariant subsets of $Y$. Suppose that $\mu(P)>0$, where $P$ is the set of all periodic points of $\left\{T_{t}\right\}$. Then $\mu(P(\leq t))>0$ for some $t>0$. By ergodicity of $\left\{T_{t}\right\}$ it follows that $\mu(P(\leq t))=1$ if $\mu(P(\leq t)) \neq 0$. Let $t_{0}=$ inf $\{t>0: \mu(P(\leq t))=1\}$. Then $\mu\left(P\left(t_{0}\right)\right)=$ 1. We may assume, without loss of generality, that $t_{0}>0$. Let $A$ be a Borel subset of $Y$ such that $0<\mu(A)<1$. Then the set $A \cap P\left(t_{0}\right)$ is invariant under $T_{t_{0}}$ and $0<\mu\left(A \cap P\left(t_{0}\right)\right)<1$. This contradicts the total ergodicity of $\mu$.

We may summarize results of this section as follows.

THEOREM 2. For every $n \geq 0$ there exists a probability measure $\mu$ defined on the $\sigma$-algebra of Borel subsets of $C^{n}(\mathbb{R}, \mathbb{R})$ satisfying the following conditions:

(a) $\mu$ is invariant under $\left\{T_{t}\right\}$;

(b) $\mu(U)>0$ for each non-empty open set $U$;

(c) $\left\{T_{t}\right\}$ is a K-flow on $\left(C^{n}(\mathbb{R}, \mathbb{R}), \mathscr{B}\left(C^{n}(\mathbb{R}, \mathbb{R})\right), \mu\right)$;

(d) $\mu$ is non-trivial;

(e) $\mu$ is a Gaussian measure.

THEOREM 3. Let $\left(X, S_{1}\right)$ be the flow defined in $\$ 2$. Then there exists a probability measure $m$ defined on $\mathscr{B}(X)$ such that $\left(X, \mathscr{B}(X), m, S_{t}\right)$ satisfies the conditions (a)-(d) of theorem 2. Moreover, if $b(x, u)$ is of the form $b(x, u)=f(x)+g(x) u$ and $U_{2}=\mathbb{R}$, then $m$ is a Gaussian measure.

Proof. According to theorem 1 there exists a homeomorphism $Q$ between $X$ and $C^{n}(\mathbb{R}, \mathbb{R})$ such that $Q \circ S_{t}=T_{t} \circ Q$. Thus, we can define a measure $m$ on $\mathscr{B}(X)$ by $m(A)=\mu(Q(A))$. The conditions (a), (b), (c) and (d) are a direct consequence of theorem 2. If $b(x, u)=f(x)+g(x) u$ and $U_{2}=\mathbb{R}$, then $Q$ is of the form $Q v=L v+v_{0}$, where $L$ is a linear isomorphism from $X$ onto $C^{n}(\mathbb{R}, \mathbb{R})$. Thus, according to theorem $2(\mathrm{e})$, the measure $m$ is Gaussian.

COROllary 2 (chaos). The flow $\left\{S_{t}\right\}$ satisfies the following two conditions:

(a) every point $v \in X$ is unstable;

(b) there exists $v \in X$ such that the trajectory of $v$ is dense in $X$.

Remark 2. The construction of the measure $m$ given in the proof of theorem 3 may be repeated as well replacing $\xi_{x}^{0}$ by $\xi_{\lambda x}^{0}$ with arbitrary $\lambda>0$. It is interesting that the measures $m_{\lambda_{1}}$ and $m_{\lambda_{2}}$ corresponding to different $\lambda_{1}$ and $\lambda_{2}$ are mutually singular.

Remark 3. Let $\zeta_{x}, x \in U_{1}$, be the process given by the formula $\zeta_{.}(\omega)=Q^{-1} \xi_{\cdot}^{n}(\omega)$. Then $m(A)=P(\zeta \in A)$ for each Borel subset $A$ of $X$. From remark 1 it follows that $\zeta_{x}, x \in U_{1}$ is a Markov process for $n=0$. 


\section{REFERENCES}

[1] J. Auslander \& J. Yorke. Interval maps, factors of maps and chaos. Tôhoku Math. J. II. Ser., 32 (1980), 177-188.

[2] P. Brunovský. Notes on chaos in the cell population partial differential equation. Nonlinear Analysis 7 (1983), 167-176.

[3] P. Brunovský \& J. Komornik. Ergodicity and exactness of the shift on $C[0, \infty)$ and the semiflow of a first order partial differential equation. To appear.

[4] A. L. Dawidowicz. On existence of invariant measure for the dynamical system generated by partial differential equation. Ann. Polon. Math. XXXIX (1983), 129-137.

[5] A. Lasota. Invariant measures and a linear model of turbulence. Rend. Sem. Mat. Univ. Padova 61 (1979), 40-48.

[6] A. Lasota. Stable and chaotic solutions of a first order partial differential equation. Nonlinear Analysis 5 (1981), 1181-1193. 